\title{
Clinical and microbiological features of vulvovaginitis in Mexican girls*
}

\author{
Mario I. Ortiz ${ }^{1,2 \#}$, Edna J. Arreola-Bautista ${ }^{3}$, Beatriz A. Sánchez-Reyes ${ }^{3}$, Georgina Romo-Hernández ${ }^{3}$, \\ Marco A. Escamilla-Acosta ${ }^{3}$ \\ ${ }^{1}$ Área Académica de Medicina del Instituto de Ciencias de la Salud de la Universidad Autónoma del Estado de Hidalgo, Pachuca, \\ Hidalgo, Mexico \\ ${ }^{2}$ Universidad del Futbol y Ciencias del Deporte, San Agustín Tlaxiaca, Hidalgo, Mexico \\ ${ }^{3}$ Hospital del Niño DIF Hidalgo, Pachuca, Hidalgo, Mexico \\ Email: \#mario_i_ortiz@hotmail.com
}

Received 21 December 2012; revised 24 January 2013; accepted 1 February 2013

\begin{abstract}
Study Objective: To determine the prevalence of vulvovaginitis, predisposing factors, microbial etiology and therapy in patients treated at the Hospital del Niño DIF, Pachuca, Hidalgo, Mexico. Design. This was an observational and descriptive study from 2006 to 2009. Setting: Hospital del Niño DIF, Pachuca, Hidalgo, Mexico. Participants. Patients from 0 to 16 years, with vulvovaginitis and/or vaginal discharge were included. Interventions: None. Main Outcome Measures: Demographic data, etiology, clinical features, risk factors and therapy were analyzed. Results: Four hundred twenty seven patients with diagnosis of vulvovaginitis were included. The average prevalence to 4 years in the study period was $0.19 \%$. The age group most affected was schoolchildren (225 cases: $52.69 \%)$. The main signs and symptoms presented were leucorrhea $(99.3 \%)$, vaginal hyperemia $(32.6 \%)$, vulvar itching (32.1\%) and erythema (28.8\%). Identified risk factors were poor hygiene $(\mathbf{1 5 . 7 \% )}$ ), urinary tract infection (14.7\%), intestinal parasites $(5.6 \%)$ and obesity or overweight (3.3\%). The main microorganisms found in vaginal cultures were enterobacteriaceae (Escherichia coli, Klebsiella and Enterococcus faecalis), Staphylococcus spp, and Gardnerella vaginalis. Several inconsistent were found in the drug prescription of the patients. Conclusion: Vulvovaginitis prevalence in Mexican girls is low and this was caused mainly by opportunist microorganisms. The initial treatment of vulvovaginitis must include hygienic measure and an antimicrobial according to the clinical features and microorganism found.
\end{abstract}

Keywords: Vulvovaginitis; Prevalence; Etiologic Microorganisms; Mexican Children and Adolescents;

\footnotetext{
"Conflict of interest: The authors of the present manuscript state that no conflict of interest exists.

\#Corresponding author.
}

\section{Therapeutic}

\section{INTRODUCTION}

Vulvovaginitis is an inflammation of the vulva and vaginal tissues [1-3]. Signs and symptoms include irritation and erythema of the vulva, vaginal discharge, soreness, itching, dysuria, and bleeding [4-7]. Vulvovaginitis is generally considered to be the commonest gynecological problem in prepubertal girls [1-3]. These girls have several predisposing factors. The anatomical factors include lack of protective labial fat pads or pubic hair, the closeness of the vulva to the anal region, and the labia minor are smaller, do not meet in midline and further tend to open up as the child squats, uncovering the most sensitive tissues within the hymeneal ring [1-3,5-9]. The physiological factors accepted consist of the thinness of the vulvar skin and physiological atrophy of the vaginal epithelium, low in glycogen, with neutral $\mathrm{pH}$ and nonproduction of cervical mucus and the poor local immune system [1-7]. All these factors make it a good culture media for the bacteria, which can easily penetrate the thin, vaginal mucosa [10-14]. Another risk factor is the poor hygiene. Children are usually careless about hand washing, often slide off toilet seats, wiping from back to front after bowel movements, therefore contaminating the vagina with enteric pathogens, scratching with dirty fingernails and playing in sand [1-9]. Other factors include the obesity, hot weather, wearing synthetic underwear, tights, slacks, using perfumed soaps, bubble bath or masturbation can exacerbate the problem [7-11]. A diffusion of bacteria from the upper airways is also possible through auto-inoculation or occasionally hematic spread. Finally, the possibility that vulvovaginitis may be secondary to intravaginal foreign bodies or to sexual abuse must also be borne in mind [1-10].

Prior to the rupture of membranes in utero, the fetal vagina is sterile, but during vaginal delivery the skin, 
anus, vagina, and oropharynx, becomes colonized with microbes [5-7,11]. The normal vaginal microflora includes gram positive and grand negative anaerobes and gram positive aerobes. The main causative agents of pediatric vulvovaginitis are represented by Streptococcus $B$ hemolytic A group, Haemophilus influenzae, and Enterobius vermicularis [5-7,11-15]. However, the non-specific infections are most common cause of premenarche vulvovaginitis, mainly due to poor perianal hygiene, with other contributing factors mentioned above. There are non-infectious causes of vulvovaginitis such as polyps and tumors, vulvar skin diseases, trauma, foreign body, etc. [1-7,11].

Although vulvovaginitis is the most common gynecological disorder seen in pediatric practice, the incidence real in Mexico is unknown. A study evaluated vulvovaginal symptoms in Mexican adolescents denying sexual activity [15]. Of 258 adolescents with vulvovaginitis, $53(20.5 \%)$ had a sexually transmitted microorganism. The presence of sexually transmitted infections was associated with lower abdominal pain, abnormally colored vaginal discharge, a positive urine culture, and abdominal ultrasonography evidence, compatible with pelvic inflammatory disease. The microorganism found were Chlamydia trachomatis (43.4\%), Trichomonas vaginalis (20.8\%), Papillomavirus (17.0\%), Gardnerella vaginalis (11.3\%) and Neisseria gonorrhoeae (7.5\%) [15]. However, authors did not research or report the not-sexually transmitted microorganism. Therefore the main objective of this study was to determine the prevalence of vulvovaginitis, its predisposing factors and microbial etiology in patients treated at the Hospital del Niño DIF, Pachuca, Hidalgo, Mexico.

\section{MATERIALS AND METHODS}

We performed a descriptive and transversal study to investigate the prevalence and clinical and microbiological features of vulvovaginitis in Mexican girls aged from 1 month to 16 years who were admitted at the Hospital del Niño DIF, Pachuca Hidalgo, Mexico, from January 2006 to December 2009. The study was approved by the Ethics and Investigation Committees and carried out according to the guidelines delineated by the Declaration of Helsinki.

Data were collected from charts of the Hospital from all girls presenting a diagnosis of infectious vulvovaginitis or vaginal discharge (the main inclusion criterion). Patients with onset of sexual activity, with a history or suspicion of sexual abuse and estrogen treatments for other conditions were excluded. The data included sociodemographic, clinical data, isolated microorganism, drugs prescribed and details of duration and presence of vulvar symptoms including pain, redness, itching, dis- charge and urinary symptoms. The medical records were reviewed by a pediatric gynecologist and a pediatric infectious, who were standardized to identify eligibility criteria and to retrieve information.

Data were entered into a computerized database. SPSS version 17 for Windows (SPSS Inc., Chicago, IL, USA) was used for descriptive statistical analyses.

\section{RESULTS}

According to information reported in the clinical records from the Hospital, 445 patients were diagnosed as "vulvovaginitis" in the years under study. All the records were analyzed according to the inclusion criteria for this study. Of the 445 cases, only 427 met the criteria for inclusion in the study period. The distribution of the number of cases each year was: 132 cases in 2006, 80 cases in 2007, 79 cases in 2008 and 136 cases in 2009. According the age ranges of the girls, 5 (1.2\%) patients had from 1 month to 23 months, 152 (35.6\%) girls from 24 months to 5 years, 225 (52.7\%) patients from 6 to 12 years and $45(10.5 \%)$ girls more than 12 years. The average age \pm SEM of all patients was $9.5 \pm 2.5$ years.

The clinical archives service reported a total of 229,568 outpatients during the 4 years of study. The average prevalence to 4 years in the study period was $0.19 \%$ (from $0.216 \%$ in $2006,0.131 \%$ in $2007,0.148 \%$ in 2008 and $0.249 \%$ in 2009).

In regard to the clinical features, Table 1 shows the signs and symptoms of the patients at the time of going to the hospital. According to the time of evolution, 286 (67\%) patients had less than six month, 90 (21\%) patients had from 6 to 24 months and 51 (12\%) patients had more than 24 moths. Table 2 shows the potential risk factors for developing vulvovaginitis.

Of all patients, only to 57 were offered interconsultations, 39 (68.4\%) of gynecology, 11 (19.3\%) of the infectious diseases service, 4 (7.0\%) of the endocrinology service, 2 (3.5\%) of the allergy service and $1(1.8 \%)$ of the psychology service.

Microorganisms were isolated in vaginal secretions of $188(44.03 \%)$ girls. Of the 188 positive cultures, there were $166(88.3 \%)$ reports with a single microorganism and $22(11.7 \%)$ reports with two or three cultured microorganisms. In Tables $\mathbf{3}$ and $\mathbf{4}$ are the main microorganisms cultivated.

In regard to the treatment of patients, sitz baths were prescribed to 87 patients $(20.4 \%)$ and toilet hygiene measures were indicated to 67 patients $(15.7 \%)$. It is noteworthy that the cleanliness was not a constant indication to all patients, even when we observed that the lack of vulvar hygiene was one of the main risk factors found in the study. With regard to treatment with antimicrobials, the data showed that they were prescribed to 
Table 1. Signs and/or symptoms that presented the patients.

\begin{tabular}{lcc}
\hline Symptoms and signs & $\mathrm{n}$ & $\%$ \\
\hline Leucorrhoea & 424 & 99.3 \\
Vaginal hyperemia & 139 & 32.6 \\
Itching & 137 & 32.1 \\
Vulvar erythema & 123 & 28.8 \\
Dysuria & 80 & 18.7 \\
Abdominal pain & 43 & 10.1 \\
Urinary frequency & 13 & 3.0 \\
Fever & 11 & 2.6 \\
Nausea and vomiting & 9 & 2.1 \\
Tenesmus & 7 & 1.6 \\
Vulvalgia & 7 & 1.6 \\
Vulvar dermatoses & 7 & 1.6 \\
Vulvar excoriations & 6 & 1.4 \\
Tenesmus urinary & 5 & 1.2 \\
Cellulite vulvar & 5 & 0.9 \\
Intestinal constipation & 4 & 0.7 \\
Vulvar stench & 3 & 0.5 \\
Back pain & 2 \\
Vaginal bleeding introit & 5.2 \\
\hline & & \\
\hline
\end{tabular}

Table 2. Risk factors presented by the patients.

\begin{tabular}{lcc}
\hline Conditions & $\mathrm{n}$ & $\%$ \\
\hline Poor perianal hygiene & 67 & 15.7 \\
Urinary tract infection & 63 & 14.7 \\
Intestinal parasites & 24 & 5.6 \\
Overweight and obesity & 14 & 3.3 \\
Upper respiratory infection & 14 & 3.3 \\
Intestinal constipation & 4 & 0.9 \\
Psychomotor retardation & 2 & 0.5 \\
Diabetes mellitus & 2 & 0.5 \\
Foreign body (paper) & 2 & 0.5 \\
Genital trauma & 2 & 0.5 \\
Vaginal septum & 1 & 0.2 \\
Lingerie adjusted & 1 & 0.2 \\
Allergic dermatitis & 1 & 0.2 \\
Attention deficit & 1 & 0.2 \\
Diarrheal syndrome & 1 & 0.2 \\
\hline
\end{tabular}

Table 3. Microorganisms present in 166 cultures.

\begin{tabular}{|c|c|c|}
\hline & $\mathrm{n}$ & $\%$ \\
\hline Escherichia coli & 39 & 23.5 \\
\hline Coagulase negative Staphylococcus & 22 & 13.3 \\
\hline Klebsiella pneumoniae & 21 & 12.7 \\
\hline Gardnerella vaginalis & 15 & 9.0 \\
\hline Enterococcus faecalis & 14 & 8.4 \\
\hline Klebsiella oxytoca & 8 & 4.8 \\
\hline Staphylococcus epidermidis & 8 & 4.8 \\
\hline Staphylococcus aureus & 7 & 4.2 \\
\hline Proteus mirabilis & 5 & 3.0 \\
\hline Shigella spp & 4 & 2.4 \\
\hline Proteus vulgaris & 3 & 1.8 \\
\hline Coagulase positive Staphylococcus & 3 & 1.8 \\
\hline Candida albicans & 3 & 1.8 \\
\hline Citrobacter freundii & 2 & 1.2 \\
\hline Enterococcus spp & 2 & 1.2 \\
\hline Staphylococcus saprophyticus & 1 & 0.6 \\
\hline Corynebacterium bovis & 1 & 0.6 \\
\hline Enterobacter cloacae & 1 & 0.6 \\
\hline Corynebacterium genitalium & 1 & 0.6 \\
\hline Corynebacterium spp & 1 & 0.6 \\
\hline Lactobacillus spp & 1 & 0.6 \\
\hline Listeria monocytogenes & 1 & 0.6 \\
\hline Morganella morganii & 1 & 0.6 \\
\hline Haemophilus influenzae & 1 & 0.6 \\
\hline Moraxella morgani & 1 & 0.6 \\
\hline
\end{tabular}

Table 4. Mixture of microorganisms found in 22 cultures.

\begin{tabular}{lcc}
\hline & $\mathrm{n}$ & $\%$ \\
\hline Escherichia coli + Staphylococcus & 7 & 31.8 \\
Escherichia coli + Corynebacterium genitalum & 2 & 9.1 \\
Escherichia coli + coagulase negative Staphylococcus. & 2 & 9.1 \\
Shigella spp + coagulase negative Staphylococcus. & 2 & 9.1 \\
Staph. saprophyticus + Enterococcus faecium & 1 & 4.5 \\
Escherichia coli + Staph. epidermidis & 1 & 4.5 \\
Enterobacter cloacae + coagulase negative & 1 & 4.5 \\
Staphylococcus & 1 & 4.5 \\
Escherichia coli + Enterococcus spp & 1 & 4.5 \\
Klebsiella pneumoniae + Proteus mirabilis & 1 & 4.5 \\
Corynebacterium striatum + Streptococcus anginosus & & \\
Escherichia coli + coagulase negative & 1 & 4.5 \\
Staphylococcus. + Strep. agalactiae & 1 & 4.5 \\
Escherichia coli + Klebsiella sp & 1 & 4.5 \\
Escherichia coli + Proteus vulgaris &
\end{tabular}


239 patients (56\%), either as monotherapy or multitherapy (2, 3 or more). In regard to monotherapy, 183 patients (76.6\% of 239 patients) received only 1 antibiotic. Table 5 shows the antimicrobial agents used as monotherapy. On the other hand, only 56 patients $(23.4 \%$ of 239 patients) received 2 or more antimicrobial consecutively (see Table 6).

\section{DISCUSSION}

While vulvovaginitis is the most common gynecological disorder seen in pediatric practice, the world prevalence is unknown. Jones reported an annual incidence of the diagnosis of vulvovaginitis in children of 1 in 10,000 patients in Plymouth, UK [8]. On the other hand, a prospective and longitudinal study in Dominic Republic reported a prevalence of vulvovaginitis of $3.69 \%$ or 369 cases by 10,000 outpatients [16]. In the present study, cases of vulvovaginitis and vaginal discharge, accounted for $0.18 \%$ of prevalence or 18 in 10,000 outpatient pediatrics. The differences between these data are due probably to the kind of study (retrospective versus prospective), the age of the patient (children and/or adolescents), and the clinical settings.

Vulvovaginitis is one of the most common disorders in pediatric gynecology. It causes distress and may be associated with a foreign body or sexual abuse. Among the neonatal period and puberty, the vaginal mucosa is atrophic due to estrogen deficiency, similar to postmenopausal vaginal mucosa. The skin is thin, lacks cornification and the $\mathrm{pH}$ is alkaline, which results to be susceptible to infection. Furthermore, the anus is near the vulva, lack of labial fat pads or pubic hair, small labia minor, lack of hygiene, and the tendency for girls to groom themselves forward, contribute to fecal contamination of the vulva [1-10]. Likewise, other risk factors are the spread of respiratory bacteria from hand to perineum and local irritants such as bubble baths, perfumed soaps, tight-fitting clothes and nylon underwear [1-10]. It is suggested that the bad habit of cleanliness, is the most common cause of vulvovaginitis in childhood. Other causes include foreign bodies, parasites, chemical irritants and acute bacterial infections. In our series, we found 12 risk factors identified, the main, poor genital hygiene (67 cases), followed by urinary tract infection (with 63 cases), intestinal parasites (24 cases), obesity (14 cases) and upper respiratory infection (14 cases) as the most significant. In general, these risk factors are agreed with the factors reported in other publications [1-10].

According the specific bacteria to produce vulvovaginitis, Haemophilus influenzae and Streptococcus pyogenes have been reported as causal agents [11-18]. These microorganisms can be transmitted from the upper respiratory tract. In our study, we did not found Streptococcus
Table 5. Antimicrobials prescribed as monotherapy to patients with vulvovaginitis.

\begin{tabular}{lcc}
\hline Drug & $\mathrm{n}$ & $\%$ \\
\hline Cefuroxime p.o. & 24 & 13.1 \\
Metronidazole p.o. & 23 & 12.6 \\
Ketoconazole cream & 19 & 10.4 \\
Clindamycin p.o. & 17 & 9.3 \\
Ampicillin p.o. & 12 & 6.6 \\
Miconazole cream & 12 & 6.6 \\
Cefixime p.o. & 10 & 5.5 \\
Amikacin i.m. & 10 & 5.5 \\
Clotrimazole cream & 7 & 3.8 \\
Gentamicin cream & 7 & 3.8 \\
Nitrofurantoin p.o. & 6 & 3.3 \\
Mupirocin cream & 5 & 2.7 \\
Amoxicillin p.o. & 4 & 2.2 \\
Nystatin cream & 4 & 2.2 \\
Dicloxacillin p.o. & 3 & 1.6 \\
Cephalexin p.o. & 3 & 1.6 \\
Other & 9.3 \\
\hline
\end{tabular}

Table 6. Antimicrobials prescribed in combination to patients with vulvovaginitis.

\begin{tabular}{lcc}
\hline Drugs & $\mathrm{N}$ & $\%$ \\
\hline Trimethoprim + sulfamethoxazole p.o. & 23 & 41.1 \\
Amoxicillin-Clavulanic Acid p.o. & 11 & 19.6 \\
$\begin{array}{l}\text { Trimethoprim + sulfamethoxazole } \\
\text { + metronidazole p.o. }\end{array}$ & 7 & 12.5 \\
$\begin{array}{l}\text { Trimethoprim + sulfamethoxazole } \\
\text { p.o. + miconazole gel }\end{array}$ & 3 & 5.4 \\
Metronidazole cream + ketoconazole cream & 2 & 3.6 \\
Ketoconazole cream + Cefuroxime p.o. & 2 & 3.6 \\
Ampicillin + Metronidazole p.o. & 2 & 3.6 \\
Cefuroxime + Metronidazole p.o. & 1 & 1.8 \\
Ketoconazole cream + Tinidazole p.o. & 1 & 1.8 \\
Clindamycin cream + Miconazole p.o. & 1 & 1.8 \\
Fluconazole p.o. + Miconazole cream & 1 & 1.8 \\
Clindamycin cream + Miconazole p.o. & 1 & 1.8 \\
Clotrimazole/gentamicin/betamethasone cream & 1 & 1.8 \\
\hline
\end{tabular}

pyogenes in the cultures and $H$. influenzae was found only in one culture. In the present study, the main op- 
portunistic pathogen microorganisms cultivated were $E$. coli (39 cases) and Coagulase negative staphylococcus (22 cases). Contrary to other publications [2,17,18], we found Klebsiella pneumoniae in 21 girls and Klebsiella oxytoca in 8 patients. These gram negative microorganisms are found in the normal flora of the mouth, skin, and intestines. Klebsiella usually causes urinary tract infections, pneumonia, and other infections in hospitalized persons whose immunity is compromised by underlying diseases, such as diabetes mellitus [19]. It is very probable that the vulvovaginitis by Klebsiella in these patients was acquired by an infectious focus elsewhere in the body.

Gardnerella vaginalis was found in 15 girls, which are associated with sexual activity or abuse [1-5,15-18]. In our setting, the pediatrician performs the examination and questioning of children or adolescents always in the presence of parents or family. For this reason, it is common that children and adolescents deny sexual activity or abuse, or any distressing, annoying and uncomfortable situation. In this case, physician must insist on investigating sexual abuse or activity in such patients.

Enterococcus faecalis was obtained in 14 cases and Proteus mirabilis in 5 cases, which together with $E$. coli (39 cases) are opportunistic bacteria frequently present in vaginal cultures. The poor or wrong hygiene of genitals contaminate the vulva and vagina with enteric nonpathogens and pathogens. In the present study, we found three cases of Candida albicans. It is important to mention that candida is rare in atrophic vagina, although many doctors believe that it is the most common cause. In general, it is difficult to determine the role of potentially pathogenic microorganisms in the production of vulvovaginitis in these patients, therefore scientific research on the etiology and treatment of genital infections must be continue and multidisciplinary.

The signs and symptoms present in this study were similar to that reported in other series [1-7]. On the other hand, we did not find a useful classification according the time of evolution. Pierce and Hart classified the vulvovaginitis as "acute" when the time of evolution is minor or equal than 6 months and "chronic" when the period of evolution is more than 6 months [1]. In our study, 181 cases $(67.03 \%)$ presented an evolution minor than 6 months, 57 (21.11\%) patients had an evolution from 6 months to 2 years and 32 (11.85\%) girls over 2 years. Therefore, it is necessary to implement a useful classification of vulvovaginitis according the evolution time and to include the cases of vulvovaginitis of repetition.

It is important to remember that the normal vaginal flora is a dynamic ecosystem in movement, and total knowledge is not yet complete, so we must be careful in the interpretation of the clinical findings and the results of cultures. As in most of the situations no cause for the non-specific vulvovaginitis is found, the initial management should be symptomatic and also depends upon eliminating the likely precipitating factor. Initial treatment should include hygienic measures and avoid contact with irritating chemicals. After that, offered antimicrobial according to the probability of the age of the patient, clinical features, and the isolation and sensitivity of the microorganisms cultivated. In the present study, there were several inconsistent in the prescription of drugs to the patients. For example, Candida albicans was found in three patients, however, fifty four antifungal drugs were prescribed to the patients. In another example, amikacin was administered to 10 patients and gentamicin to seven patients. There is enough evidence that shows the nephrotoxicity and ototoxicity produced by aminoglycoside therapy [20]. For this reason, the use of aminoglycosides for the treatment of vulvovaginitis is not justified.

In conclusion, vulvovaginitis prevalence in Mexican girls is low and this was caused mainly by opportunist microorganisms. The initial treatment of vulvovaginitis must include hygienic measure and an antimicrobial according to the clinical features and microorganism found. It is necessary to establish clinical guides to diagnostic and management vulvovaginitis in children and adolescents in all the clinical settings.

\section{REFERENCES}

[1] Pierce, A.M. and Hart, C.A. (1992) Vulvovaginitis: Causes and management. Archives of Disease in Childhood, 6, 509-512. doi:10.1136/adc.67.4.509

[2] Stricker, T., Navratil, F. and Sennhauser, F.H. (2003) Vulvovaginitis in prepubertal girls. Archives of Disease in Childhood, 88, 324-326. doi:10.1136/adc.88.4.324

[3] Jaquiery, A., Stylianopoulos, A., Hogg, G. and Grover, S. (1999) Vulvovaginitis: Clinical features, aetiology, and microbiology of the genital tract. Archives of Disease in Childhood, 81, 64-67. doi:10.1136/adc.81.1.64

[4] Joishy, M., Ashtekar, C.S., Jain, A. and Gonsalves, R. (2005) Do we need to treat Vulvovaginitis in prepubertal girls? British Medical Journal, 330, 186-188. doi:10.1136/bmj.330.7484.186

[5] Jasper, J.M. (2009) Vulvovaginitis in the prepubertal child. Clinical Pediatric Emergency Medicine, 10, 10-13. doi:10.1016/j.cpem.2009.01.003

[6] Fischer, G. and Rogers, M. (2000) Vulvar disease in children: A clinical audit of 130 cases. Pediatric Dermatology, 17, 1-6. doi:10.1046/j.1525-1470.2000.01701.x

[7] Dei, M., Di Maggio, F., Di Paolo, G. and Bruni, V. (2010) Vulvovaginitis in childhood. Best Practice \& Research, Clinical Obstetrics \& Gynaecology, 24, 129-137. doi:10.1016/j.bpobgyn.2009.09.010

[8] Jones, R. (1996) Childhood Vulvovaginitis and vaginal discharge in general practice. Family Practice, 13, 369372. doi:10.1093/fampra/13.4.369 
[9] Merkley, K. (2005) Vulvovaginitis and vaginal discharge in the pediatric patient. Journal of Emergency Nursing, 31, 400-402. doi:10.1016/j.jen.2005.04.023

[10] Richens, J. (1994) Sexually transmitted diseases in children in developing countries. Genitourinary Medicine, 70, 278-283.

[11] Sharma, B., Preston, J. and Greemwood, P. (2004) Management of vulvovaginitis and vaginal discharge in prepubertal girls. Reviews in Gynaecological Practice, 4, 111-120. doi:10.1016/j.rigp.2003.12.005

[12] Rees, A. and Mott, A. (2001) Management of vaginal discharge in pre-pubertal children. Current Paediatrics, 11, 375-380. doi:10.1054/cupe.2001.0205

[13] Cox, R.A. and Slack, M.P.E. (2002) Clinical and microbiological features of Haemophilus influenza vulvovaginitis in young girls. Journal of Clinical Pathology, 55, 961-964. doi:10.1136/jcp.55.12.961

[14] Cox, R.A. (1997) Haemophilus influenza: An underrated cause of vulvovaginitis in young girls. Journal of Clinical Pathology, 50, 765-768. doi:10.1136/jcp.50.9.765

[15] Velarde-Jurado, E., Estrada-Reyes, E., Eraña-Guerra, L., Raya-Rivera, A., Velázquez-Armenta, E.Y. and NavaOcampo, A.A. (2003) Sexually transmitted infections as- sociated with vulvovaginal symptoms in adolescents denying sexual activity. Salud Pública de México, 45, S641S646. doi:10.1590/S0036-36342003001100009

[16] Rodríguez Pita, N.M., Pérez, J.M., Gutiérrez, M.C., Paulino, R.H., Rojas, J. and Chestaro, L. (2003) Prevalence of vulvovaginitis in children under 15 years. $R e$ vista Médica de Dominicana, 64, 48-50.

[17] Steele, A.M. and de San Lazaro, C. (1994) Transhymeal cultures for sexually transmissible organisms. Archives of Disease in Childhood, 71, 423-427. doi:10.1136/adc.71.5.423

[18] Hammerschlag, M.R., Alpert, S., Rosner, I., Thurston, P., Semine, D., McComb, D., et al. (1978) Microbiology of the vagina in children: Normal and potentially pathogenic organisms. Pediatrics, 62, 57-62.

[19] Podschun, R. and Ullmann, U. (1998) Klebsiella spp. as nosocomial pathogen: Epidemiology, taxonomy, typing methods, and pathogenicity factors. Clinical Microbiology Reviews, 11, 589-603.

[20] Ariano, R.E., Zelenitsky, S.A. and Kassum, D.A. (2008) Aminoglycoside-induced vestibular injury: Maintaining a sense of balance. The Annals of Pharmacotherapy, 42, 1282-1289. doi:10.1345/aph.1L001 